Original Paper http://ajol.info/index.php/ijbcs http://indexmedicus.afro.who.int

\title{
Caractérisation des performances zootechniques de la population de tilapia du Nil (Oreochromis niloticus) du lac de la vallée du Kou (Burkina Faso)
}

\author{
Rokyatou SISSAO ${ }^{1,2^{*}}$, Morgan Paul Magouana ANVO $^{3}$ et Aboubacar TOGUYENI ${ }^{1,4}$ \\ ${ }^{1}$ Unité de Recherche Aquaculture et Biodiversité Aquatique / Laboratoire d'Etudes et de Recherche sur les \\ Ressources Naturelles et Sciences de l'Environnement / Université Nazi BONI, 01 BP 1091 Bobo-Dioulasso \\ 01, Burkina Faso. \\ ${ }^{2}$ Institut de l'Environnement et de Recherches Agricoles / Centre National de la Recherche Scientifique et \\ Technologique, 01 BP 910 Bobo-Dioulasso 01, Burkina Faso. \\ ${ }^{3}$ Station de Recherche en Pêche et Aquaculture Continentale / Centre National de Recherche Agronomique, 01 \\ BP 633 Bouaké, Côte d'Ivoire. \\ ${ }^{4}$ Centre International de Recherche-Développement sur l'Elevage en zone Subhumide, 01 BP 454 Bobo- \\ Dioulasso 01, Burkina Faso. \\ *Auteur correspondant ; E-mail : sissaorokyatou@gmail.com
}

\section{REMERCIEMENTS}

Cette étude a été réalisée dans le cadre du projet de recherche-développement intitulé "Développement d'une filière de production de semences améliorées de poissons, tilapia du Nil (Oreochromis niloticus) et poisson chat Africain (Clarias gariepinus) ». Nous exprimons notre gratitude à l'UEMOA (Union Economique et Monétaire Ouest Africain) qui a financé ce projet.

\section{RESUME}

Les performances zootechniques des souches indigènes sont un facteur déterminant pour leur valorisation dans les systèmes d'élevage locaux. La présente étude avait pour objectif d'analyser celles de la population de tilapia du Nil du lac de la vallée du Kou. L'approche méthodologique a consisté à analyser les performances initiales de 6 familles non apparentées, d'alevins de 10 jours post-fécondation (jpf) dans un dispositif en duplicata. Une seconde expérimentation a porté sur 6 autres familles de juvéniles monosexes mâles avec un poids moyen d'environ $100 \mathrm{~g}$, qui ont été marqués individuellement. Les taux de survie des alevins (10 à 90 jpf) varient de 70 à $95 \%$ et aucune mortalité n'a été enregistrée pendant la phase de grossissement. La croissance initiale est caractérisée par un taux variant de 15,62 à 9,62\% par jour (10 à 40 jpf) et de 5,15 à 9,21\% par jour (40 à 90 jpf) en fonction de la famille. De même, le gain de poids quotidien des lots monosexes mâles varie de 1,03 à 1,46 g par jour. Au regard de ces résultats préliminaires, une souche locale d'aquaculture peut être développée à partir des familles et des individus les plus performants de cette population.

(C) 2019 International Formulae Group. All rights reserved.

Mots clés : Tilapia du Nil, aquaculture, souches indigènes, lac de la vallée du Kou, croissance. 


\title{
Characterization of the zootechnical performances of Lake Kou Nile tilapia (Oreochromis niloticus) population (Burkina Faso)
}

\begin{abstract}
The zootechnical performances of indigenous strains are determinant for their valorization in local aquaculture systems. This study was conducted in order to analyze under controlled conditions, the growth and survival performances of the Lake Kou Nile tilapia population. Two experiments were performed using 12 fullsib families. In the first experiment, duplicate groups of 6 families were used from 10 to 90 days' postfertilization (dpf). Six groups of all-males tagged juveniles having a mean body weight of $100 \mathrm{~g}$, were used in the second experiment. Survival rates (10 to $90 \mathrm{dpf}$ ) ranged from 70 to $95 \%$ and no mortality was recorded during the grow-out period. In the first experiment, the specific growth rate varied from 15.62 to $9.62 \%$ per day (10 to $40 \mathrm{dpf}$ ) and from 9.21 to $5.15 \%$ per day (40 to $90 \mathrm{dpf}$ ). The daily weight gains of all-males families varied from 1.03 to $1.46 \mathrm{~g}$ per day. These preliminary results, indicate that a local aquaculture strain could be developed, using the best performing families and individuals of this population.
\end{abstract}

(C) 2019 International Formulae Group. All rights reserved.

Keywords: Nile tilapia, aquaculture, indigenous strains, lake Kou, growth.

\section{INTRODUCTION}

L'offre mondiale de produits d'origine aquatique estimée à 171 millions de tonnes en 2016 provient majoritairement de la pêche qui fournit $53,18 \%$ de la production totale. Cependant la baisse des prises halieutiques a entrainé une stagnation puis une diminution considérable des produits issus de la pêche de capture. A l'opposé l'aquaculture se développe au rythme rapide de $13 \%$ par an, et est devenue depuis 2014 la principale source d'approvisionnement de poissons destinés à la consommation humaine (FAO, 2018). Néanmoins, des pistes d'amélioration de la production aquacole mondiale sont toujours explorées, afin que ce secteur puisse satisfaire durablement la forte demande en produits aquatiques, générée par la forte croissance démographique (Diana, 2009; FAO, 2018)

Plus de 360 espèces de poissons sont élevées dans le monde dont les tilapias qui constituent le deuxième groupe d'espèces le plus élevé (Chevassus-au-Louis et Lazard, 2009 ; Fontaine et al., 2009 ; FAO, 2018). Le tilapia du Nil, Oreochromis niloticus $(O$. niloticus) est la principale espèce de tilapia élevée et la quatrième espèce aquacole mondiale, eu égard à ses aptitudes piscicoles remarquables: croissance rapide, résistance aux maladies et aux manipulations, reproduction en captivité, qualité organoleptique et nutritionnelle de la chair et valeur commerciale. De plus, il s'adapte à différents systèmes d'élevage et son régime alimentaire très peu exigeant en protéines est un facteur d'optimisation des coûts de production (Chevassus-au-Louis et Lazard, 2009 ; Fontaine et al., 2009; Lazard, 2009 ; Amoussou et al., 2017). Ainsi, il est aujourd'hui élevé dans plus de 130 pays dont principalement la Chine qui fournit la majorité de sa production totale estimée à 4,2 millions de tonnes en 2016 (FAO, 2018). Paradoxalement, l'Afrique qui est le continent d'origine de cette espèce, est un grand importateur de tilapia du Nil (Gordon et al., 2013).

De façon générale, il y a un réel déséquilibre géographique de l'aquaculture mondiale, se traduisant par la domination de l'Asie qui assure près de $90 \%$ de la production mondiale, et la Chine seule contribue à hauteur de 61,5\% (FAO, 2018). La production africaine est quasiment insignifiante et représente seulement $2,5 \%$ de la production mondiale. Ainsi, $73 \%$ des poissons consommés en Afrique sont importés, ce qui impacte négativement sur l'accessibilité des produits halieutiques et en conséquence une diminution de la consommation de poisson qui est inférieur à $10 \mathrm{~kg}$ par habitant et par an (FAO, 2018). Le développement de l'aquaculture africaine est donc à la fois un enjeu économique et de sécurité alimentaire et nutritionnelle.

Ce développement ne saurait être durable que s'il est basé sur l'élevage des espèces indigènes, ce qui nécessite leur 
domestication et une amélioration génétique afin d'obtenir des souches performantes. Or, actuellement, c'est plutôt l'utilisation des souches performantes exotiques qui est privilégiée dans la plupart des pays, induisant une dépendance des producteurs et surtout une menace grave pour la biodiversité locale (IUCN, 2007; FAO, 2010). Ainsi, pour éviter les risques de pollution des ressources génétiques locales, le développement de souches d'élevage adaptées au contexte écologique local est recommandé en lieu et place des introductions de souches exogènes (IUCN, 2007; Fontaine et al., 2009).

C'est dans ce cadre qu'en Afrique de l'Ouest, la souche Akossombo a été développée à partir des populations de $O$. niloticus du bassin de la Volta. De même la souche Bouaké a été obtenue à partir des populations des bassins du Nil et de la Volta. En effet, l'analyse de la variabilité intraspécifique des stocks naturels peut conduire à la mise en évidence de souches ayant des potentialités aquacoles supérieures à celles des souches d'élevage (Buschmann et al., 2006). Par ailleurs, il est aussi bien établi qu'au sein d'une même population, ces performances peuvent varier considérablement à l'échelle de la famille et même de l'individu (Chevassusau-Louis et Lazard, 2009). Il est donc important de caractériser les performances zootechniques de plusieurs populations naturelles en tenant compte des différents niveaux de variabilité (Fontaine et Le Bail, 2004 ; Chevassus-au-Louis et Lazard, 2009).

C'est dans ce contexte que cette étude a été initiée pour analyser les performances zootechniques de la population de $O$. niloticus du lac de la vallée du Kou (lac Kou), une retenue d'eau du bassin de la Volta, située à l'Ouest du Burkina Faso. Les paramètres zootechniques de 12 familles non apparentées ont ainsi été évaluées en milieu contrôlé, afin d'analyser la variabilité inter et intrafamiliale de cette population.

\section{MATERIEL ET METHODES Géniteurs}

L'étude a porté sur la population sauvage de $O$. niloticus du lac de la vallée du Kou (Figure 1). Ce lac, d'une superficie d'environ 100 ha, est situé dans la partie Ouest du Burkina Faso à $11^{\circ} 23^{\prime}$ de latitude
Nord et $4^{\circ} 24^{\prime}$ de longitude Ouest (Sanogo et al., 2014). Les géniteurs au nombre de 91 y ont été capturés puis transférés à la station d'expérimentation piscicole de l'Unité de Recherche Aquaculture et Biodiversité Aquatique de l'Université Nazi BONI (UR$\mathrm{ABAQ} / \mathrm{UNB}$ ). Le stock de géniteurs était constitué de 46 mâles et 45 femelles qui avaient un poids moyen de $484,95 \mathrm{~g}$ et $254 \mathrm{~g}$ respectivement. Les mâles et les femelles ont été stockés séparément dans deux bassins hors sol de $7,2 \mathrm{~m}^{3}$, en circuit ouvert et soumis aux conditions naturelles de température et de photopériode. Ils ont ensuite été marqués individuellement à l'aide de transpondeur de type PIT-Tag (passive integrated transponder Tag) de la marque EasyTrac-ID.

\section{Obtention des alevins}

Les alevins ont été obtenus par reproduction semi-artificielle. Les pontes et les fécondations ont été réalisés en aquariums de 300 litres montés en circuit fermé dans une enceinte close. Un mâle et trois femelles ont été sélectionnés dans le stock de géniteurs et maintenus dans un aquarium de 300 litres. Après une ponte et fécondation naturelles, les œufs ont été récupérés de la cavité buccale de la femelle, et incubés dans des bouteilles McDonald de 2 litres chacun, en circuit fermé. Chaque descendance obtenue a été référencée grâce aux identifiants (code PIT-Tag de 15 chiffres) du couple de géniteurs. Au total 12 descendances non apparentées, issues de 12 mâles et 12 femelles ont été générées pour les expérimentations.

\section{Matériel de mesure}

La température, le $\mathrm{pH}$ et le taux d'oxygène dissout de l'eau, ont été contrôlés l'aide du multiparamètre $(H A C H)$. Le poids a été déterminé à l'aide d'une balance de précision $0,001 \mathrm{~g}(K E R N)$. Les longueurs totale et standard ont été déterminées à l'aide d'un ichtyomètre (précision $1 \mathrm{~mm}$ ).

Expérimentation 1 : suivi des performances zootechniques pendant le prégrossissement de 10 à 90 jpf

Cette première expérimentation a consisté en un suivi des performances zootechniques de six familles non apparentées au cours de la phase d'alevinage allant de 10 à 
90 jours post-fécondation (jpf). Au début (10 jpf), un échantillon de 30 alevins a été pesé globalement pour évaluer le poids moyen initial. L'expérimentation a été réalisée en duplicata dans un circuit fermé d'aquariums de 50 litres, à une densité initiale de 2 alevins par litre (Tableau 1). Un aliment commercial (Skretting Norway) dosant $58 \%$ de protéines a été utilisé pour le nourrissage. Les alevins ont été nourris ad libitum 4 fois par jour $(9 \mathrm{~h}, 11$ $\mathrm{h}, 13 \mathrm{~h}$ et $16 \mathrm{~h}$ ).

Le dispositif expérimental était soumis à une photopériode de $12 \mathrm{~h}$ de lumière artificielle et $12 \mathrm{~h}$ d'obscurité. La température, le $\mathrm{pH}$ et le taux d'oxygène dissous ont été contrôlés 2 fois par jour et maintenus dans les intervalles propices pour la pisciculture du tilapia du Nil à $27,13 \pm 0,71{ }^{\circ} \mathrm{C}$ pour la température, $6,84 \pm 0,46$ pour le $\mathrm{pH}$ et $5,14 \pm$ $0,96 \mathrm{mg} / \mathrm{l}$ pour l'oxygène dissous (Toguyeni, 1996) durant toute l'expérimentation.

Deux pêches de contrôle ont été réalisées à 40 jpf et à 90 jpf, soit après 30 et 80 jours d'élevage respectivement. A cet effet, les poissons de chaque lot ont été dénombrés pour évaluer le taux de survie. Aussi, le poids individuel et les longueurs (totale et standard) ont été mesurés pour un échantillon de 30 alevins par lot.

\section{Expérimentation 2: suivi des performances zootechniques pendant grossissement des lots monosexes mâles}

Cette seconde expérimentation a consisté à suivre les performances zootechniques pendant la phase de grossissement (Tableau 2). Elle a été conduite pendant 3 mois, avec deux groupes composés chacun de lots monosexes mâles de même âge issus de 3 familles non apparentées. Les mâles ont été identifiés sur la base du dimorphisme sexuel de la papille urogénitale (Toguyéni, 1996; Baroiller et D'Cotta, 2018). Ainsi, 30 mâles ont été prélevés aléatoirement par famille et marqués (Tableau 2) à l'aide des transpondeurs de type PIT Tag afin d'évaluer les performances zootechniques individuelles.

L'expérimentation a été réalisée dans six bassins bétonnés de $2 \mathrm{~m}^{3}$ en circuit ouvert, en respectant une densité de 15 poissons par $\mathrm{m}^{2}$. Les poissons ont été nourris ad libitum 3 fois par jour ( $9 \mathrm{~h}, 12 \mathrm{~h}$ et $15 \mathrm{~h}$ ), avec l'aliment commercial (Skretting Norway) dosant $40 \%$ de protéines. Le dispositif expérimental a été soumis aux conditions naturelles de températures et de photopériode. Les paramètres physico-chimiques de l'eau ont été contrôlés 2 fois par jour et maintenus dans les intervalles optimums pour l'élevage de $O$. niloticus à $29,48 \pm 1,19{ }^{\circ} \mathrm{C}, 7,27 \pm 0,41$ et à $4,76 \pm 1,04 \mathrm{mg} / \mathrm{l}$ respectivement pour la température, le $\mathrm{pH}$ et l'oxygène dissous (Toguyeni, 1996).

Des pêches de contrôle ont été réalisées tous les 30 jours. Pendant ces contrôles, les poissons sont identifiés individuellement grâce aux références des PIT-Tags. Le poids individuel et les longueurs (totale et standard) ont été déterminés pour chaque individu.

\section{Indices zootechniques calculés}

Pour analyser les performances zootechniques des différents lots expérimentaux, les indices suivants ont été calculés :

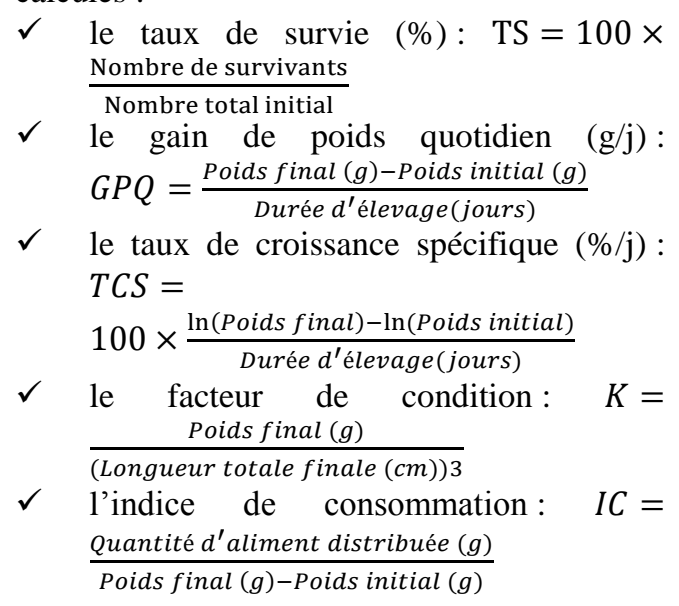

\section{Analyse statistique}

Le logiciel STATISTICA 12.0 a été utilisé pour l'analyse des données. Une analyse de variance (ANOVA) à un ou deux critères a été réalisée sur l'ensemble des données zootechniques. Le test de NewmanKeuls a permis ensuite de comparer les moyennes obtenues au seuil de probabilité de $5 \%$. Les résultats obtenus sont présentés sous la forme «moyenne \pm écart type ». 


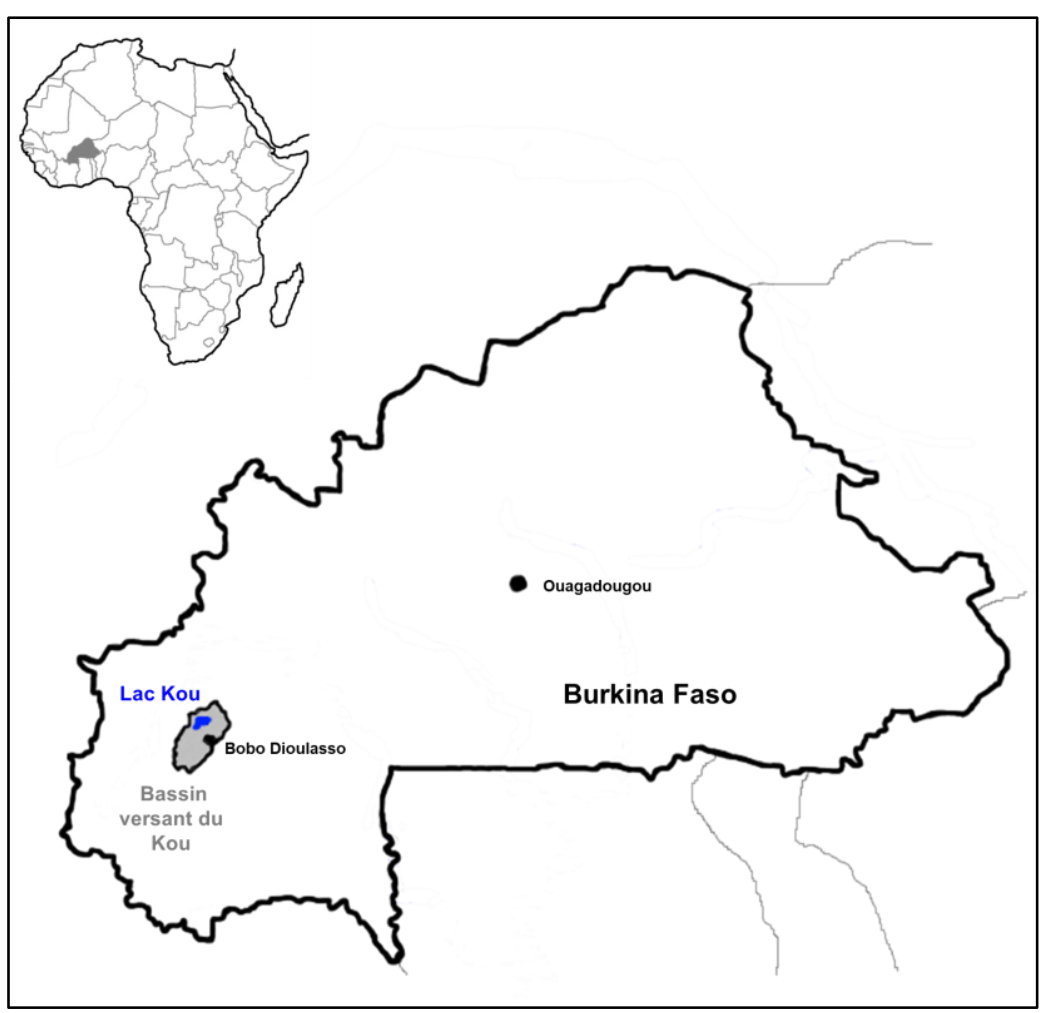

Figure 1: Localisation du lac de la vallée du Kou (Source Sissao et al., 2019). Lac Kou : lac de la vallée du Kou.

Tableau 1 : Identifiants et paramètres initiaux des familles utilisées pour le prégrossissement.

\begin{tabular}{cccccc}
\hline Familles & $\begin{array}{c}\text { Géniteurs } \\
\text { Mâle } \times \text { Femelle }\end{array}$ & Réplicats & $\begin{array}{c}\text { Poids moyen } \\
\text { initial à 10jpf } \\
\text { (g) }\end{array}$ & $\begin{array}{c}\text { Effectif initial } \\
(\text { N/réplicat })\end{array}$ & $\begin{array}{c}\text { Densité initiale } \\
\text { (alevin/litre) }\end{array}$ \\
\hline Fam.1 & M1 $\times$ F1 & 2 & 0,008 & 100 & 2 \\
Fam.2 & M2 $\times$ F2 & 2 & 0,010 & 100 & 2 \\
Fam.3 & M3 × F3 & 2 & 0,008 & 100 & 2 \\
Fam.4 & M4 × F4 & 2 & 0,007 & 100 & 2 \\
Fam.5 & M5 $\times$ F5 & 2 & 0,010 & 100 & 2 \\
Fam.6 & M6 $\times$ F6 & 2 & 0,013 & 100 & 2 \\
\hline
\end{tabular}

Fam. : Famille ; M :mâle ; F : femelle N : Nombre. 
Tableau 2 : Identifiants et paramètres initiaux des familles utilisées pour le grossissement des lots monosexes mâles.

\begin{tabular}{|c|c|c|c|c|c|}
\hline $\begin{array}{c}\text { Groupes } \\
\text { homogènes }\end{array}$ & Familles & $\begin{array}{c}\text { Géniteurs } \\
\text { Mâle } \times \text { Femelle }\end{array}$ & $\begin{array}{l}\text { Poids moyen } \\
\text { initial (g) }\end{array}$ & $\begin{array}{c}\text { Effectif } \\
(\mathbf{N} / \text { famille })\end{array}$ & $\begin{array}{c}\text { Densité } \\
\text { (indiv. } / \mathbf{m}^{2} \text { ) }\end{array}$ \\
\hline \multirow{3}{*}{ Groupe A } & Fam.7 & M7 × F7 & \multirow{3}{*}{$90,35 \pm 0,61$} & 30 & 15 \\
\hline & Fam.8 & $\mathrm{M} 8 \times \mathrm{F} 8$ & & 30 & 15 \\
\hline & Fam.9 & $\mathrm{M} 9 \times \mathrm{F} 9$ & & 30 & 15 \\
\hline \multirow{3}{*}{ Groupe B } & Fam.10 & $\mathrm{M} 10 \times \mathrm{F} 10$ & \multirow{3}{*}{$107,55 \pm 0,19$} & 30 & 15 \\
\hline & Fam.11 & $\mathrm{M} 11 \times \mathrm{F} 11$ & & 30 & 15 \\
\hline & Fam.12 & $\mathrm{M} 12 \times \mathrm{F} 12$ & & 30 & 15 \\
\hline
\end{tabular}

Fam. : Famille ; M : mâle ; F : femelles N : Nombre. Indiv. : Individus.

\section{RESULTATS}

Evolution des performances zootechniques pendant le prégrossissement $(10$ à 90 jpf) Performances zootechniques à $40 \mathrm{jpf}$

A $40 \mathrm{jpf}$, le poids moyen varie entre $0,18 \mathrm{~g}$ (famille 1 ) et $1,15 \mathrm{~g}$ (familles 5 et 6 ); la longueur standard a varié de $1,92 \mathrm{~cm}$ (famille 1) à 3,44 cm (famille 6) (Tableau 3). Les analyses statistiques n'ont toutefois pas révélé de différence significative entre les six familles $(\mathrm{P}>0,05)$ pour ces paramètres de croissance pondérale et linéaire.

Les variations moyennes du gain de poids quotidien (GPQ) et du coefficient de condition (K) moyen (Tableau 3) ne sont pas significativement différente d'une famille à une autre $(\mathrm{p}>0,05)$. Ces moyennes ont varié de 0,01 à $0,04 \mathrm{~g} / \mathrm{j}$ pour le GPQ et de 0,01 à 0,02 pour le coefficient de condition.

Par contre, pour l'indice de consommation (IC), la valeur obtenue pour la famille $1(3,14 \pm 0,01)$ est significativement différente de celles des cinq autres familles (Tableau 3).
L'analyse du taux de croissance spécifique (TCS) montre une variabilité interfamiliale significative (Tableau 3). En effet, les TCS des familles $1(9,62 \pm 0,14 \% / \mathrm{j})$ et $3(11,19 \pm 1,00 \% / \mathrm{j})$ sont significativement inférieurs $(p<0,05)$ à ceux des quatre autres familles où les taux varient entre 14,81 \pm $0,09 \% / j$ et $15,67 \pm 1,43 \% / j$ (Tableau 3 ).

Par ailleurs, les taux de survie ont varié entre $80,50 \pm 6,36 \%$ (famille 1 ) et 95,00 \pm $4,24 \%$ (famille 2). Il n'y a toutefois pas de différence significative entre les six familles ( $\mathrm{P}>0,05)$ (Tableau 3).

\section{Performances zootechniques à 90 jpf}

A ce niveau, la variabilité de la croissance pondérale est plus marquée $(12,57$ $\pm 0,62$ à $29,29 \pm 0,81 \mathrm{~g})$ et l'analyse statistique révèle des différences significatives $(\mathrm{p}<0,05)$ entre certaines familles (Tableau 3 ). Les poids corporels moyens des familles 2 et 3 sont significativement inférieurs à ceux des familles 1 et 4 , tandis que les familles 5 et 6 présentent les plus fortes croissances pondérales $(\mathrm{p}<0,05)$. 
Par contre, au niveau de la croissance linéaire, l'analyse statistique montre que les longueurs standards des familles $5(9,20 \pm$ $0,08 \mathrm{~cm})$ et $6(9,20 \pm 0,08 \mathrm{~cm})$ sont significativement supérieurs à celles des quatre autres familles $(\mathrm{p}<0,05)$.

Pour les gains de poids quotidiens moyens, une variabilité interfamiliale similaire à celle de la croissance linéaire a été observée (Tableaux 3). Les familles 5 et 6 présentent les moyennes les plus élevées, qui sont statistiquement différentes $(\mathrm{p}<0,05)$ de celles des autres familles.

Le taux de croissance spécifique, quant à lui, varie de $5,15 \pm 1,39 \% / j$ (famille 2) à $9,21 \pm 0,34 \% / j$ (famille 1 ); seules ces deux valeurs extrêmes sont significativement différentes entre elles (Tableau 3).

Aucune différence significative n'est par contre observée en ce qui concerne les indices de consommation ( $p>0,05)$. La moyenne la plus faible $(1,75 \pm 0,2)$ et celle la plus élevée $(2,33 \pm 0,15)$ ont été observées chez les familles 5 et 6 respectivement.

L'analyse statistique révèle une forte variabilité interfamiliale $(\mathrm{p}<0,05)$ pour les coefficients de condition, bien que les valeurs soient toutes très faibles, variant de 0,016 à 0,02 (Tableau 3).

Les taux de survie ne sont pas significativement différents entre les six familles (Tableau 3). Les taux enregistrés varient entre 70,00 $\pm 9,43 \%$ (famille 5) et $93,33 \pm 4,7 \%$ (familles 6 ).

\section{Evolution des performances zootechniques des lots monosexes mâles}

Les données de croissance pondérale et linéaire des lots monosexes mâles pendant la phase de grossissement sont présentées dans la Figure 2. Les deux groupes initialement constitués en fonction du poids moyen de départ sont maintenus après 30 jours de suivi aussi bien pour le poids moyen individuel que pour la longueur standard.
Par contre, à partir de 60 jours d'élevage, les performances de croissance pondérale sont plus hétérogènes et des différences significatives apparaissent entre certaines familles $(\mathrm{p}<0,05)$. Cette variabilité interfamiliale $\mathrm{du}$ poids corporel, indépendamment des paramètres initiaux, est plus prononcée à la fin de l'expérimentation (Figure 2). Ainsi les familles 8 (205 $\pm 18,04$ g) et $11(239 \pm 18,07 \mathrm{~g})$ présentent les meilleures performances de croissance respectivement pour les groupes $\mathrm{A}$ et $\mathrm{B}$ $(\mathrm{p}<0,05)$. La même tendance est observée au niveau de la croissance linéaire mais les différences ne sont pas significatives (Figure 2).

Les valeurs du coefficient de condition varient très faiblement selon la famille et les différences ne sont pas significatives (Tableau 4). Par contre, l'analyse du taux de croissance spécifique, du gain de poids quotidien et de l'indice de consommation, révèle une évolution très hétérogène selon la famille (Tableau 4). Les indices (GPQ, TCS et IC) des familles 8 et 11 sont significativement meilleurs comparativement aux autres familles de leur groupe respectif ( $p>0,05)$.

Par ailleurs, aucune mortalité n'a été enregistrée durant cette deuxième expérimentation (Tableau 4).

Les résultats révèlent aussi une forte variabilité intrafamiliale des performances de croissance pondérale individuelle (Figure 3). En effet, des individus ayant un poids initial similaire évoluent de façon très différente. Dans les six familles étudiées, certains individus n'ont pas atteint le poids final théorique attendu (illustré par la courbe de tendance linéaire), tandis que d'autres ont largement dépassé le seuil attendu (Figure 3). Dans l'ensemble les gains de poids individuels ont varié de 0,39 à $2,09 \mathrm{~g} / \mathrm{j}$ et de 0,55 à $2,14 \mathrm{~g} / \mathrm{j}$ pour les individus des groupes $\mathrm{A}$ et $\mathrm{B}$ respectivement. 
Tableau 3 : Variabilité interfamiliale des paramètres zootechniques pendant le prégrossissement.

\begin{tabular}{cccccccc} 
Paramètres & âge & Fam.1 & Fam.2 & Fam.3 & Fam.4 & Fam.5 & Fam.6 \\
\hline & $10 j p f$ & 0,008 & 0,010 & 0,008 & 0,007 & 0,010 & 0,013 \\
PM (g) & $40 j p f$ & $0,18 \pm 0,01^{\mathrm{a}}$ & $1,09 \pm 0,75^{\mathrm{a}}$ & $0,29 \pm 0,09^{\mathrm{a}}$ & $0,85 \pm 0,02^{\mathrm{a}}$ & $1,15 \pm 0,48^{\mathrm{a}}$ & $1,15 \pm 0,52^{\mathrm{a}}$ \\
& & & & & & & \\
& $90 \mathrm{jpf}$ & $17,98 \pm 2,31^{\mathrm{b}}$ & $12,57 \pm 0,62^{\mathrm{c}}$ & $13,26 \pm 1,33^{\mathrm{c}}$ & $19,14 \pm 2,38^{\mathrm{b}}$ & $29,29 \pm 0,81^{\mathrm{a}}$ & $28,36 \pm 2,79^{\mathrm{a}}$ \\
& & & & & & & \\
& $40 j p f$ & $1,92 \pm 0,00^{\mathrm{a}}$ & $3,18 \pm 0,71^{\mathrm{a}}$ & $2,20 \pm 0,22^{\mathrm{a}}$ & $2,93 \pm 0,10^{\mathrm{a}}$ & $3,33 \pm 0,43^{\mathrm{a}}$ & $3,44 \pm 0,50^{\mathrm{a}}$
\end{tabular}

LS (cm)

90jpf $\quad 7,80 \pm 0,34^{\mathrm{b}} \quad 7,08 \pm 0,11^{\mathrm{b}} \quad 7,42 \pm 0,15^{\mathrm{b}} \quad 7,85 \pm 0,35^{\mathrm{b}} \quad 9,20 \pm 0,08^{\mathrm{a}} \quad 9,63 \pm 0,48^{\mathrm{a}}$

40jpf $\quad 0,01 \pm 0,00^{\mathrm{a}} \quad 0,04 \pm 0,02^{\mathrm{a}} \quad 0,01 \pm 0,00^{\mathrm{a}} \quad 0,03 \pm 0,00^{\mathrm{a}} \quad 0,04 \pm 0,02^{\mathrm{a}} \quad 0,04 \pm 0,02^{\mathrm{a}}$

GPQ $(g / j)$

90jpf $\quad 0,36 \pm 0,05^{\mathrm{b}} \quad 0,23 \pm 0,00^{\mathrm{c}} \quad 0,26 \pm 0,02^{\mathrm{c}} \quad 0,37 \pm 0,05^{\mathrm{b}} \quad 0,56 \pm 0,03^{\mathrm{a}} \quad 0,54 \pm 0,05^{\mathrm{a}}$

40jpf $\quad 9,62 \pm 0,14^{\mathrm{b}} \quad 15,20 \pm 2,48^{\mathrm{a}} \quad 11,19 \pm 1,00^{\mathrm{b}} \quad 14,81 \pm 0,09^{\mathrm{a}} \quad 15,67 \pm 1,43^{\mathrm{a}} \quad 15,63 \pm 1,56^{\mathrm{a}}$

$\operatorname{TCS}(\% / \mathrm{j})$

90jpf $\quad 9,21 \pm 0,34^{\mathrm{a}} \quad 5,15 \pm 1,39^{\mathrm{b}} \quad 7,66 \pm 0,40^{\mathrm{ab}} \quad 6,22 \pm 0,20^{\mathrm{b}} \quad 6,56 \pm 0,91^{\mathrm{ab}} \quad 6,52 \pm 0,74^{\mathrm{ab}}$

40jpf $\quad 0,01 \pm 0,00^{\mathrm{a}} \quad 0,01 \pm 0,00^{\mathrm{a}} \quad 0,01 \pm 0,00^{\mathrm{a}} \quad 0,02 \pm 0,00^{\mathrm{a}} \quad 0,02 \pm 0,00^{\mathrm{a}} \quad 0,01 \pm 0,00^{\mathrm{a}}$

K

90 jpf $\quad 0,016 \pm 0,00^{\mathrm{c}} \quad 0,018 \pm 0,00^{\mathrm{b}} \quad 0,019 \pm 0,00^{\mathrm{b}} \quad 0,019 \pm 0,00^{\text {ba }} \quad 0,019 \pm 0,00^{\text {ba }} \quad 0,020 \pm 0,00^{\mathrm{a}}$

40jpf $\quad 3,14 \pm 0,01^{\mathrm{b}} \quad 3,04 \pm 0,03^{\mathrm{a}} \quad 3,09 \pm 0,03^{\mathrm{a}} \quad 3,02 \pm 0,00^{\mathrm{a}} \quad 3,03 \pm 0,01^{\mathrm{a}} \quad 3,04 \pm 0,02^{\mathrm{a}}$

IC

90jpf $2,14 \pm 0,05^{\mathrm{a}} \quad 2,28 \pm 0,14^{\mathrm{a}} \quad 2,09 \pm 0,16^{\mathrm{a}} \quad 2,05 \pm 0,17^{\mathrm{a}} \quad 1,75 \pm 0,20^{\mathrm{a}} \quad 2,33 \pm 0,15^{\mathrm{a}}$

40jpf $\quad 80,5 \pm 6,36^{\mathrm{a}} \quad 95,0 \pm 4,24^{\mathrm{a}} \quad 93,0 \pm 5,66^{\mathrm{a}} \quad 77,0 \pm 5,66^{\mathrm{a}} \quad 89,5 \pm 9,19^{\mathrm{a}} \quad 91,0 \pm 5,66^{\mathrm{a}}$

$\operatorname{TS}(\%)$

90jpf $\quad 88,33 \pm 2,36^{\mathrm{a}} \quad 86,67 \pm 0,0^{\mathrm{a}} \quad 85,0 \pm 7,07^{\mathrm{a}} \quad 81,67 \pm 7,07^{\mathrm{a}} \quad 70,0 \pm 9,43^{\mathrm{a}} \quad 93,33 \pm 4,71^{\mathrm{a}}$

Les valeurs représentent la moyenne \pm l'écart-type. Les valeurs de la même ligne portant des lettres différentes sont significativement différentes $(\mathrm{p}<0,05)$. PM : poids moyen ; LS : longueur standard ; GPQ : gain de poids quotidien, TCS : taux de croissance spécifique ; $\mathrm{K}$ : facteur de condition ; IC : indice de consommation et TS : taux de survie. 
A
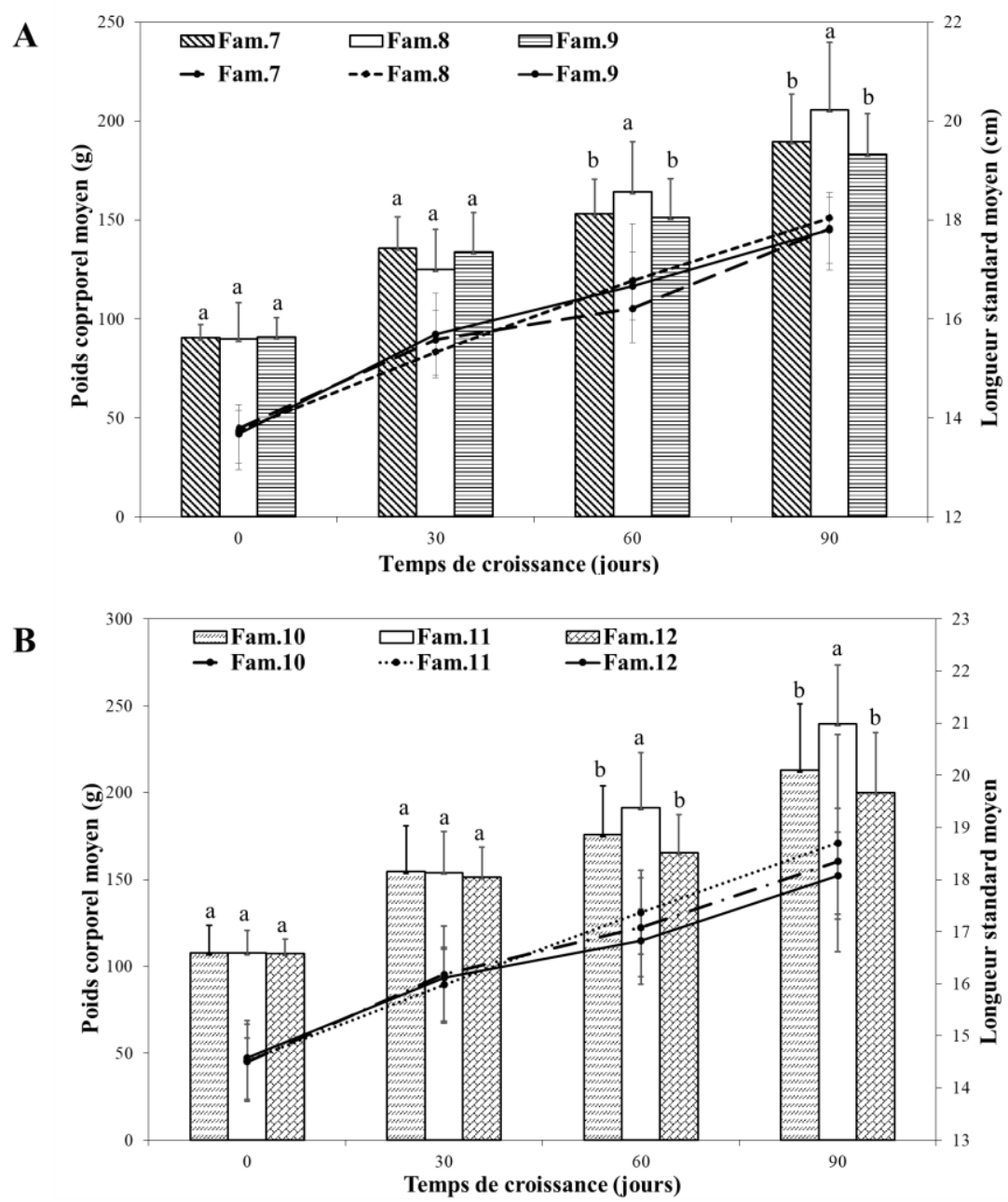

Figure 2 : Variabilité interfamiliale de la croissance pondérale et linéaire des lots monosexe mâles des groupes A et B.

Les figures présentent sous forme d'histogrammes l'évolution de la croissance pondérale de chaque famille pendant la phase de grossissement. Les résultats sont présentés à des intervalles de 30 jours. Chaque histogramme porte une barre représentant la valeur positive de l'écart type. Les histogrammes de la même période portant des lettres différentes sont significativement différents $(p<0,05)$. Les courbes montrent l'évolution moyenne de la longueur standard ; les valeurs positives et négatives de l'écart type sont représentées. Il n'y a pas de différences significatives entre les moyennes des familles pour chaque période $(\mathrm{p}>0,05)$.

Tableau 4 : Variabilité interfamiliale des paramètres zootechniques pendant le grossissement des lots monosexes mâles.

\begin{tabular}{ccccccc}
\hline Groupes & Familles & GPQ $(\mathbf{g} / \mathbf{j})$ & TCS $(\% / \mathbf{j})$ & K & IC & TS $(\%)$ \\
\hline & Fam.7 & $1,17 \pm 0,33^{\mathrm{b}}$ & $0,75 \pm 0,15^{\mathrm{b}}$ & $0,018 \pm 0,001^{\mathrm{a}}$ & $1,44 \pm 0,43^{\mathrm{b}}$ & 100 \\
Groupe A & Fam.8 & $1,46 \pm 0,35^{\mathrm{a}}$ & $0,88 \pm 0,17^{\mathrm{a}}$ & $0,019 \pm 0,001^{\mathrm{a}}$ & $1,33 \pm 0,40^{\mathrm{b}}$ & 100 \\
& Fam.9 & $1,03 \pm 0,37^{\mathrm{b}}$ & $0,68 \pm 0,18^{\mathrm{b}}$ & $0,018 \pm 0,001^{\mathrm{a}}$ & $1,96 \pm 0,86^{\mathrm{a}}$ & 100 \\
\hline
\end{tabular}




\begin{tabular}{lcccccc}
\hline & Fam.10 & $1,10 \pm 0,25^{\mathrm{b}}$ & $0,82 \pm 0,13^{\mathrm{b}}$ & $0,018 \pm 0,001^{\mathrm{a}}$ & $1,42 \pm 0,32^{\mathrm{b}}$ & 100 \\
Groupe B & Fam.11 & $1,29 \pm 0,39^{\mathrm{a}}$ & $0,93 \pm 0,25^{\mathrm{a}}$ & $0,019 \pm 0,002^{\mathrm{a}}$ & $1,30 \pm 0,41^{\mathrm{b}}$ & 100 \\
& Fam.12 & $1,03 \pm 0,24^{\mathrm{b}}$ & $0,78 \pm 0,16^{\mathrm{b}}$ & $0,017 \pm 0,001^{\mathrm{a}}$ & $1,72 \pm 0,45^{\mathrm{a}}$ & 100
\end{tabular}

Les valeurs représentent la moyenne \pm l'écart-type. Les valeurs de la même colonne portant des lettres différentes sont significativement différentes $(\mathrm{p}<0,05)$. GPQ : gain de poids quotidien, TCS : taux de croissance spécifique ; $\mathrm{K}$ : facteur de condition ; IC : indice de consommation et TS : taux de survie.
Poids individuel initial
Poids individuel final
Courbe de tendance
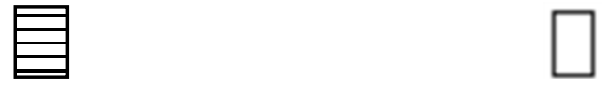
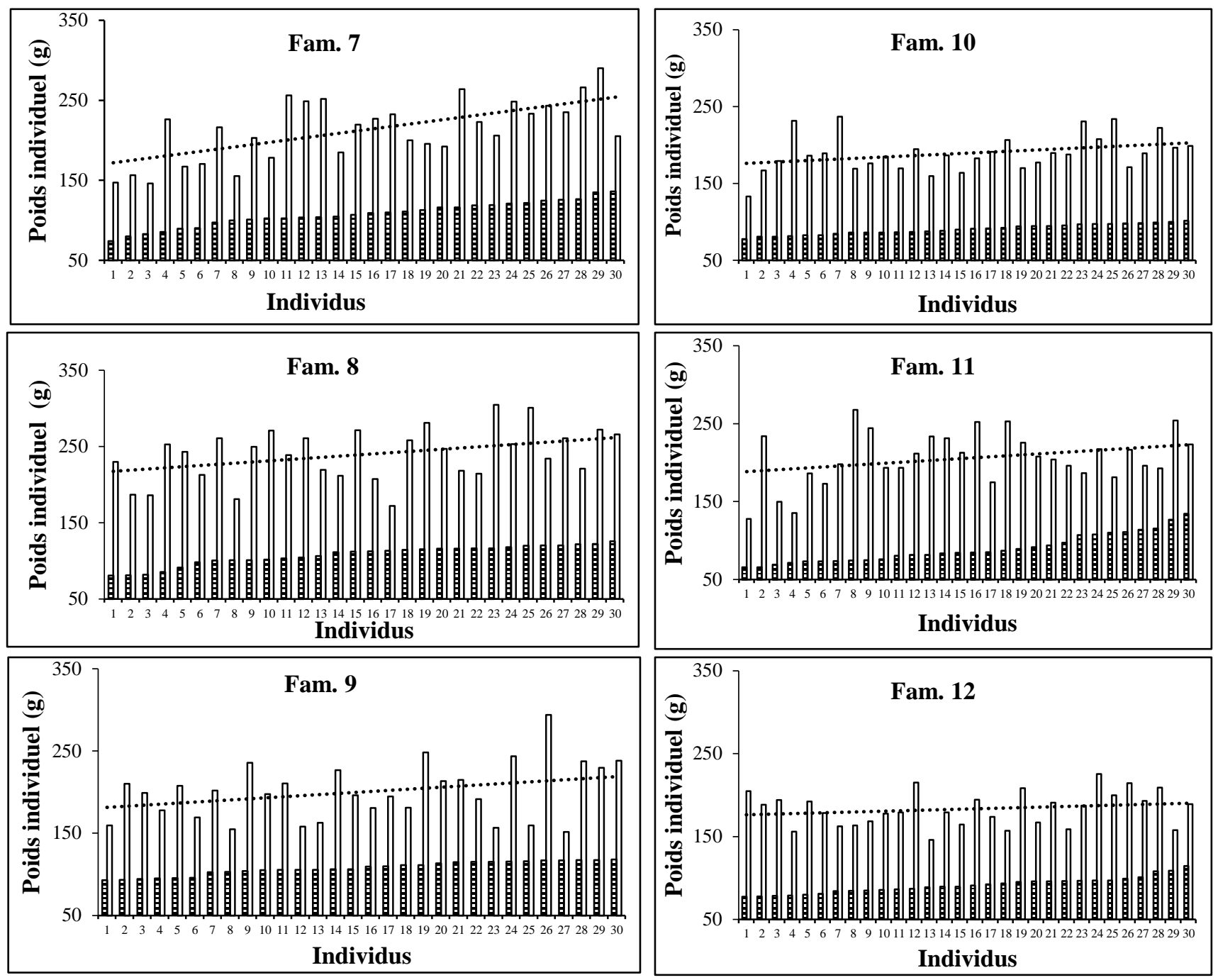

Figure 3 : Variabilité intrafamiliale de la croissance pondérale.

Les figures représentent les niveaux de croissance individuelle initiale et finale de six familles. Chaque famille est composée de 30 individus marques qui sont présentés en abscisse par ordre croissant du poids initial. La courbe de tendance linéaire représente le niveau de croissance finale théorique attendu. 


\section{DISCUSSION}

Le rôle majeur du tilapia du Nil dans l'économie aquacole mondiale résulte notamment de ses performances zootechniques, celles-ci peuvent cependant varier selon la souche et les conditions d'élevage. C'est ainsi que plusieurs travaux ont porté sur l'analyse des performances de reproduction et de croissance de différentes populations ou souches. Les résultats de la présente étude, qui a porté sur la souche Kou, ont montré que cette dernière a de bonnes performances zootechniques comparativement à certaines populations des travaux antérieurs.

En effet, les taux de survie de cette population, en cours de domestication, sont dans les mêmes intervalles observés au cours des études antérieures dont les valeurs sont comprises entre $75 \%$ et $100 \%$ quel que soit la souche ou la population étudiée (Ridha, 2006 ; Azaza et al., 2008; Bamba et al., 2008; Caldini et al., 2011 ; Sarr et al., 2016). Cela témoigne des bonnes performances de survie du tilapia du Nil, quand bien même les conditions ne sont pas optimales comme cela a été montré dans les travaux de Sifa et al., 2002.

Aussi, d'une manière générale, les performances de croissance de cette souche Kou, sont similaires voire supérieures à celles de plusieurs souches domestiques de la littérature. A 90 jpf, le taux de croissance spécifique a varié de 5,15 à $9,21 \% / \mathrm{j}$ et le gain de poids quotidien de 0,23 à $0,56 \mathrm{~g} / \mathrm{j}$ pour l'ensemble des six familles analysées. Ces valeurs sont relativement proches des intervalles 5,38 à $7,18 \% / \mathrm{j}$ et 0,10 à $0,31 \mathrm{~g} / \mathrm{j}$ obtenus pour la souche Maryut en fonction de la température de l'eau d'élevage (Azaza et al., 2008). Par contre, elles sont significativement supérieures à celles de la souche Brésilienne qui a un taux oscillant entre 2,5 et $2,94 \% / \mathrm{j}$ selon le mode de nourrissage (Caldini et al., 2011). Comparativement à la souche Suprême $(0,52$ $\mathrm{g} / \mathrm{j}$ ), les performances sont similaires quand la température de l'eau est à $22^{\circ} \mathrm{C}$. Par contre, le gain de poids de cette souche à $28{ }^{\circ} \mathrm{C}$ (température optimale) est significativement supérieur $(5,46 \mathrm{~g} / \mathrm{j})$ (Santos et al., 2013).
Aussi, les gains de poids des souches Red et GIFT, avec respectivement 2,37 et $6,89 \mathrm{~g} / \mathrm{j}$ (Santos et al., 2013), se révèlent supérieurs pendant la même phase. Cependant, l'étude de Santos et al. (2013) a été réalisée sur des lots monosexes mâles issus d'un traitement hormonal à la $17 \alpha$-méthyltestostérone, qui a un effet anabolisant (Robles Basto et Mena Herrera, 2013). Par ailleurs, il faut signaler que les résultats des souches Maryut (Azaza et al., 2008), Brésilienne (Caldini et al., 2011) et Suprême (Santos et al., 2013) ont été obtenus dans des conditions expérimentales similaires à celle de la présente étude. En effet, ces travaux précédents (Azaza et al., 2008; Caldini et al., 2011; Santos et al., 2013) ont été réalisés en circuit fermé où les poissons ont été nourris avec de l'aliment performant dosant 40 à $45 \%$ de protéines.

Pendant le grossissement des lots monosexes mâles, des performances de 1,03 à $1,46 \mathrm{~g} / \mathrm{j}$ (GPQ) et de 0,68 à $0,93 \% / \mathrm{j}$ (TCS) ont été enregistrées pour l'ensemble des 6 familles analysées. Comparativement aux résultats obtenus dans des infrastructures expérimentales similaires et un poids initial identique $(100 \mathrm{~g})$, les performances des lots monosexes mâles de la population du lac Kou sont relativement similaires à celles de la souche améliorée GIFT (Ridha et al., 2006). En effet, les résultats de cette souche améliorée de l'ordre de $0,97 \% / \mathrm{j}$ et $1,5 \mathrm{~g} / \mathrm{j}$ se situent dans les intervalles de croissance obtenus pour la souche Kou. Toutefois Ridha et al. (2006) ont enregistré ces résultats en élevage mixte, à forte densité et en utilisant un aliment dosant $35 \%$ de protéines.

La comparaison des performances de croissance des différentes familles non apparentées utilisées a révélé une forte variabilité interfamiliale. Le suivi individuel des poissons marqués a révélé également une forte variabilité intrafamiliale. Cette hétérogénéité de croissance observée à 90 jpf, puis pendant la phase de grossissement des lots monosexes mâles est un phénomène couramment rencontré chez les poissons (Chevassus-au-Louis et Lazard, 2009; Compaoré et al., 2017). Il résulterait soit des facteurs inhérents à l'espèce, soit des 
conditions d'élevage (Kestemont et al., 2003). Toutefois, l'homogénéité de nos conditions d'élevage suggère que les facteurs abiotiques n'ont pas été un facteur influençant l'expression des performances zootechniques des poissons élevés. En effet, au niveau de la qualité physico-chimique de l'eau, les moyennes des paramètres contrôlés se situent dans les intervalles propices pour la pisciculture du tilapia du Nil (Tran-Duy et al., 2008). D'autre part, les faibles valeurs du coefficient de condition $(<2)$ et de l'indice de consommation des différents lots, indiquent aussi des conditions d'élevage optimales (van de Nieuwegiessen et al., 2009).

Par conséquent, l'hétérogénéité de croissance inter et intrafamiliale mise en évidence ici, résulterait plutôt des variations génétiques ou des changements morphophysiologiques inhérents à l'espèce $O$. niloticus comme cela a été montré au cours de plusieurs travaux (Kestemont et al., 2003; Fontaine et Le Bail, 2004). Chez cette espèce, le dimorphisme sexuel de croissance est le principal facteur morpho-physiologique d'hétérogénéité (Phelps et Popma, 2000; Beardmore et al., 2001; Baroiller et D'Cotta, 2018). Ce différentiel de croissance qui apparaît à la maturité sexuelle, résulterait des différences physiologiques et éthologiques entre les mâles et les femelles (Phelps et Popma, 2000 ; Beardmore et al, 2001; Robles Basto et Mena Herrera, 2013; Baroiller et D'Cotta, 2018). Toutefois, ces mécanismes de la croissance différentielle liée au sexe ne peuvent pas être à l'origine de l'hétérogénéité de croissance de la population du lac de la vallée du Kou, car l'étude a porté sur des lots mixtes sexuellement immatures (expérimentation 1) et sur des lots monosexes mâles matures (expérimentation 2). Les différences de croissance observées seraient donc liées à la variabilité des potentialités intrinsèques des différentes familles (Compaoré et al., 2017) car les performances de croissance sont un caractère héritable contrôlé par des facteurs génétiques (Mandiki et al., 2004; Chevassus-au-Louis et Lazard, 2009). Par ailleurs, au-delà de l'effet de l'ascendance, les potentialités de croissance dépendent également des individus en présence, et sont susceptibles de varier selon la combinaison allélique transmise par les deux parents à chaque individu (Fontaine et Le Bail, 2004). La variabilité inter et intrafamiliale des performances de croissance de la population du lac de la vallée du Kou, peut être judicieusement exploitée pour le développement d'un programme d'amélioration devant aboutir à la sélection d'une souche plus performante pour l'aquaculture locale (Little et Hulata, 2000). Cette sélection peut se faire par une approche génomique en identifiant les marqueurs génétiques de croissance, soit par une méthode empirique consistant à sélectionner les individus les plus performants à chaque génération (Ponzoni et al., 2005; Vandeputte et al., 2009).

\section{Conclusion}

L'objectif de cette étude était de caractériser les performances zootechniques de la population de tilapia du Nil du lac de la vallée du Kou. Les résultats obtenus indiquent des potentialités zootechniques très prometteuses pour cette population sauvage en cours de domestication. L'analyse des paramètres zootechniques de 12 familles non apparentées révèle des indices de performances relativement similaires à celles des souches domestiques améliorées. Nos résultats mettent aussi en évidence une variabilité inter et intrafamiliale significative, dont la prise en compte permettra d'optimiser tout programme de sélection et d'amélioration basée sur la population de $O$. niloticus du lac de la vallée du Kou.

\section{CONFLIT D'INTERETS}

Tous les auteurs ont lu et approuvé la version finale de l'article. Les auteurs déclarent qu'il n'y a aucun conflit d'intérêts lié à cette étude et à l'utilisation des données.

\section{CONTRIBUTIONS DES AUTEURS}

RS a rédigé le protocole, réalisé les expérimentations, analysé les données et rédigé l'article. AMPM a participé à la rédaction de l'article. AT est le superviseur 
scientifique de l'étude: conception et réalisation du design expérimental, correction approfondie du protocole de recherche et de l'article.

\section{REMERCIEMENTS}

Nous remercions Téné R. Kombéré et Marc-henry S. Gandon ainsi que les autres stagiaires et le personnel de l'unité de recherche en aquaculture et biodiversité aquatique qui ont participé à la collecte des données. Nous remercions également Henri Gildas Sanou qui a capturé et fourni les géniteurs du lac de la vallée du Kou.

\section{REFERENCES}

Amoussou TO, Toguyeni A, Toko II, Chikou A, Karim IYA. 2017. Caractéristiques biologiques et zootechniques des tilapias africains Oreochromis niloticus (Linnaeus, 1758) et Sarotherodon melanotheron (Rüppell, 1852): une revue. Int. J. Biol. Chem. Sci., 10(4): 1869-1887.

DOI: http://dx.doi.org/10.4314/ijbcs.v10i4.35

Azaza MS, Dhraïef MN, Kraïem MM. 2008. Effects of water temperature on growth and sex ratio of juvenile Nile tilapia Oreochromis niloticus (Linnaeus) reared in geothermal waters in southern Tunisia. Journal of Thermal Biology, 33: 98-105.

DOI: http://dx.doi.org/10.1016/j.jtherbio.2007. 05.007

Bamba Y, Ouattara A, Moreau J, Gourène G. 2007. Apports relatifs en nourritures naturelle et artificielle dans l'alimentation du tilapia Oreochromis niloticus en captivité. Bulletin Français de la Pêche et de la Pisciculture, 386: 55-68.

DOI: http://dx.doi.org/10.1051/kmae:2007012

Baroiller J-F, D'Cotta H. 2018. Sex Control in Tilapias. In Sex Control in Aquaculture, Wang H-P, Piferrer F, Chen S-L, Shen Z-G (ed). John Wiley \& Sons Ltd: Chichester, UK; 189-234. DOI: http://dx.doi.org/10.1002/978111912729 1.ch9
Beardmore JA, Mair GC, Lewis RI. 2001. Monosex male production in finfish as exemplified by tilapia: applications, problems, and prospects. Aquaculture, 197: 283-301. DOI: http://dx.doi.org/10.1016/B978-0-44450913-0.50015-1

Buschmann AH, Riquelme VA, HernándezGonzález MC, Varela D, Jiménez JE, Henríquez LA, Vergara PA, Guíñez R, Filún L. 2006. A review of the impacts of salmonid farming on marine coastal ecosystems in the southeast Pacific. ICES Journal of Marine Science, $\mathbf{6 3}$ : 1338-1345.

DOI: http://dx.doi.org/10.1016/j.icesjms.2006. 04.021

Caldini NN, Rebouças VT, Cavalcante DDH, Martins RB, Sá MVDC. 2011. Water quality and Nile tilapia growth performance under different feeding schedules. Acta Sci. Anim. Sci., 33: 427430.

DOI: http://dx.doi.org/10.4025/actascianimsci. v33i4.12207

Chevassus-au-Louis B, Lazard J. 2009. Perspectives pour la recherche biotechnique en pisciculture. Agriculture, 2: 91-96. DOI: http://dx.doi.org/10.1684/agr.2009.0289

Compaore I, Toguyeni A, Rougeot C, Kestemont P, Melard C. 2017. Etude comparative des performances zootechniques d'une souche domestiquée de Clarias gariepinus (Burchell, 1822) et d'une souche sauvage de Clarias anguillaris (Linnaeus, 1758). Int. J. Biol. Chem. Sci., 10(5): 2138-2150. DOI: http://dx.doi.org/10.4314/ijbcs.v10i5.16

Dan NC, Little DC. 2000. The culture performance of monosex and mixed-sex new-season and overwintered fry in three strains of Nile tilapia (Oreochromis niloticus) in northern Vietnam. Aquaculture, 184: 221-231. DOI: http://dx.doi.org/10.1016/S00448486(99)00329-4

Diana JS. 2009. Aquaculture Production and Biodiversity Conservation. BioScience, 59: 27-38.

DOI: 
http://dx.doi.org/10.1525/bio.2009.59.1. 7

FAO. 2010. Développement de l'aquaculture : gestion des ressources génétiques. Directives techniques pour une pêche responsable. Organisation des Nations Unies pour l'Alimentation et l'Agriculture, Rome, 160p.

FAO. 2018. La situation mondiale des pêches et de l'aquaculture 2018. Atteindre les objectifs de développement durable. Organisation des Nations Unies pour l'Alimentation et l'Agriculture, Rome, $237 \mathrm{p}$.

Fontaine P, Le Bail P-Y. 2004. Domestication et croissance chez les poissons. INRA Productions Animales, 17(3): 217-225.

Fontaine P, Legendre M, Vandeputte M, Fostier A. 2009. Domestication de nouvelles espèces et développement durable de la pisciculture. Agriculture, 18(2-3): $\quad 1196124 . \quad$ DOI: http://dx.doi.org/10.1684/agr.2009.0293

Gordon A, Fine gold C, Crissman CC, Pulis A. 2013. Fish production, consumption and trade in Sub-Saharan Africa: A Review analysis. In International Working Paper "Sub-Saharan Africa Fish Trade in a Changing Climate". WorldFish ; 48p.

IUCN. 2007. Guide pour le développement durable de l'aquaculture méditerranéenne: interactions entre l'aquaculture et l'environnement. Union internationale pour la conservation de la nature et de ses ressources, Gland et Malaga, 110p.

Kestemont P, Jourdan S, Houbart M, Mélard C, Paspatis M, Fontaine P, Cuvier A, Kentouri M, Baras E. 2003. Size heterogeneity, cannibalism and competition in cultured predatory fish larvae: biotic and abiotic influences. Aquaculture, 227: 333-356. DOI: http://dx.doi.org/10.1016/S00448486(03)00513-1

Lazard J. 2009. La pisciculture des tilapias. Agriculture, 18(2-3): 174-182. DOI: http://dx.doi.org/10.1684/agr.2009.0305.
Little DC, Hulata G. 2000. Strategies for tilapia seed production. In Tilapias: Biology and Exploitation, Malcolm CM. Beveridge B, McAndrew J (ed). Fish and Fisheries Series; 267-326.

Mandiki SNM, Blanchard G, Mélard C, Koskela J, Kucharczyk D, Fontaine P, Kestemont P. 2004. Effects of geographic origin on growth and food intake in Eurasian perch (Perca fluviatilis L.) juveniles under intensive culture conditions. Aquaculture, 229: 117-128.

DOI: http://dx.doi.org/10.1016/S00448486(03)00359-4

Phelps RP, Popma TJ. 2000. Sex reversal of tilapia. In Tilapia Aquaculture in the Americas, Costa-Pierce BA, Rakocy JE (ed). The World Aquaculture Society: Baton Rouge, Louisiana, United States; 34-59.

Ponzoni RW, Hamzah A, Tan S, Kamaruzzaman N. 2005. Genetic parameters and response to selection for live weight in the GIFT strain of Nile tilapia (Oreochromis niloticus). Aquaculture, 247: 203-210. DOI: http://dx.doi.org/10.1016/j.aquaculture.2 005.02 .020

Ridha MT. 2006. Comparative study of growth performance of three strains of Nile tilapia, Oreochromis niloticus, L. at two stocking densities. Aquaculture Research, 37: 172-179. DOI: http://dx.doi.org/10.1111/j.13652109.2005.01415.x

Robles Basto CM, Mena Herrera A. 2013. Somatic growth effects of intramuscular injection of growth hormone in androgen-treated juvenile Nile tilapia, Oreochromis niloticus (Perciformes: Cichlidae). Rev. Biol. Trop., 61(1): 203212.

DOI: http://dx.doi.org/10.15517/rbt.v61i1.109 95

Sanogo S, Kabre J, Cecchi P. 2014. Inventaire et distribution spatio-temporelle des macroinvertébrés bioindicateurs de trois plans d'eau du bassin de la Volta au Burkina Faso. Int. J. Biol. Chem. Sci., 
8(3):

1005-1029.

DOI:

http://dx.doi.org/10.4314/ijbcs.v8i3.16

Santos VB, Mareco EA, Silva MDP. 2013. Growth curves of Nile tilapia (Oreochromis niloticus) strains cultivated at different temperatures. Acta Scientiarum Animal Sciences, 35: 235242.

DOI:

http://dx.doi.org/10.4025/actascianimsci. v35i3.19443

Sarr SM, Thiam A, Faye EH, Sene M, Ndiaye M. 2016. Production d'alevins de Tilapia (Oreochromis niloticus) avec 3 aliments à base de sous-produits agro-industriels au Nord du Sénégal. Int. J. Biol. Chem. Sci., 9(5): 2598-2606. DOI: http://dx.doi.org/10.4314/ijbcs.v9i5.29

Sifa L, Chenhong L, Dey M, Gagalac F, Dunham R. 2002. Cold tolerance of three strains of Nile tilapia, Oreochromis niloticus. China. J. Aquaculture, 213: 123-129.

DOI:

http://dx.doi.org/10.1016/S00448486(02)00068-6

Sissao R, D’Cotta H, Baroiller J-F, Toguyeni A. 2019. Mismatches between the genetic and phenotypic sex in the wild Kou population of Nile tilapia Oreochromis niloticus. Peer J., 7: e7709. DOI:

http://dx.doi.org/10.7717/peerj.7709
Toguyéni A. 1996. La croissance différentielle liée au sexe chez le Tilapia (Pisces: Cichlidae), Oreochromis niloticus (Linnaeus, 1758): contribution des facteurs génétiques, nutritionnels, comportementaux, et recherche d'un relais endocrinien. Thèse de doctorat unique, Université de Rennes 1, Rennes, $158 \mathrm{p}$.

Tran-Duy A, Schrama JW, van Dam AA, Verreth JAJ. 2008. Effects of oxygen concentration and body weight on maximum feed intake, growth and hematological parameters of Nile tilapia, Oreochromis niloticus. J. Aquaculture, 275: 152-162. DOI: http://dx.doi.org/10.1016/j.aquaculture.2 007.12 .024

van de Nieuwegiessen PG, Olwo J, Khong S, Verreth JAJ, Schrama JW. 2009. Effects of age and stocking density on the welfare of African catfish, Clarias gariepinus Burchell. J. Aquaculture, 288: $69-75 . \quad$ DOI: http://dx.doi.org/10.1016/j.aquaculture.2 008.11.009

Vandeputte M, Baroiller J-F, Haffray P, Quillet E. 2009. Amélioration génétique des poissons: quelles réalisations et quels défis pour demain? Agriculture, 18(2): 3262-269.

DOI: http://dx.doi.org/10.1684/agr.2009.0291. 